\title{
Treatment and outcomes of arthrogryposis in the upper extremity
}

\author{
Scott Oishi $^{1}$ (1) | Olga Agranovich ${ }^{2}$ | Dan Zlotolow ${ }^{3}$ | Lindley Wall ${ }^{4}$ | Chris Stutz ${ }^{1}$ | \\ Giorgio Pajardi $^{5}$ | Chiara Novelli ${ }^{5}$ | Hisham Abdel Ghani ${ }^{6}$ | Andrea Jester ${ }^{7}$ | \\ Carley Vuillermin $^{8}$ | Michelle James ${ }^{9}$ | Mary C. Manske ${ }^{9}$ | Terri Beckwith ${ }^{1}$
}

\author{
${ }^{1}$ Texas Scottish Rite Hospital for Children, \\ Dallas, Texas \\ ${ }^{2}$ The Turner Scientific Research Institute for \\ Children's Orthopedics, Saint Petersburg, \\ Russia \\ ${ }^{3}$ Shriners Hospitals for Children, Philadelphia, \\ Pennsylvania \\ ${ }^{4}$ Department of Orthopedic Surgery, \\ Washington University, Saint Louis, Missouri \\ ${ }^{5}$ Department of Clinical Sciences and \\ Community Health, The University of Milan, \\ Milan, Italy \\ ${ }^{6}$ Kasr Al Ainy Hospital, Faculty of Medicine, \\ Cairo University, Cairo, Egypt \\ ${ }^{7}$ Department of Surgery, Indiana University, \\ Indianapolis, Indiana \\ ${ }^{8}$ Boston Children's Hospital, Harvard Medical \\ School, Boston, Massachusetts \\ ${ }^{9}$ Shriners Hospital for Children Northern \\ California, Sacramento, California

\section{Correspondence} \\ Scott Oishi, Center for Excellence in Hand \\ Disorders, Texas Scottish Rite Hospital for \\ Children, 2222 Welborn Street, Dallas, TX \\ 75219. \\ Email: scott.oishi@tsrh.org
}

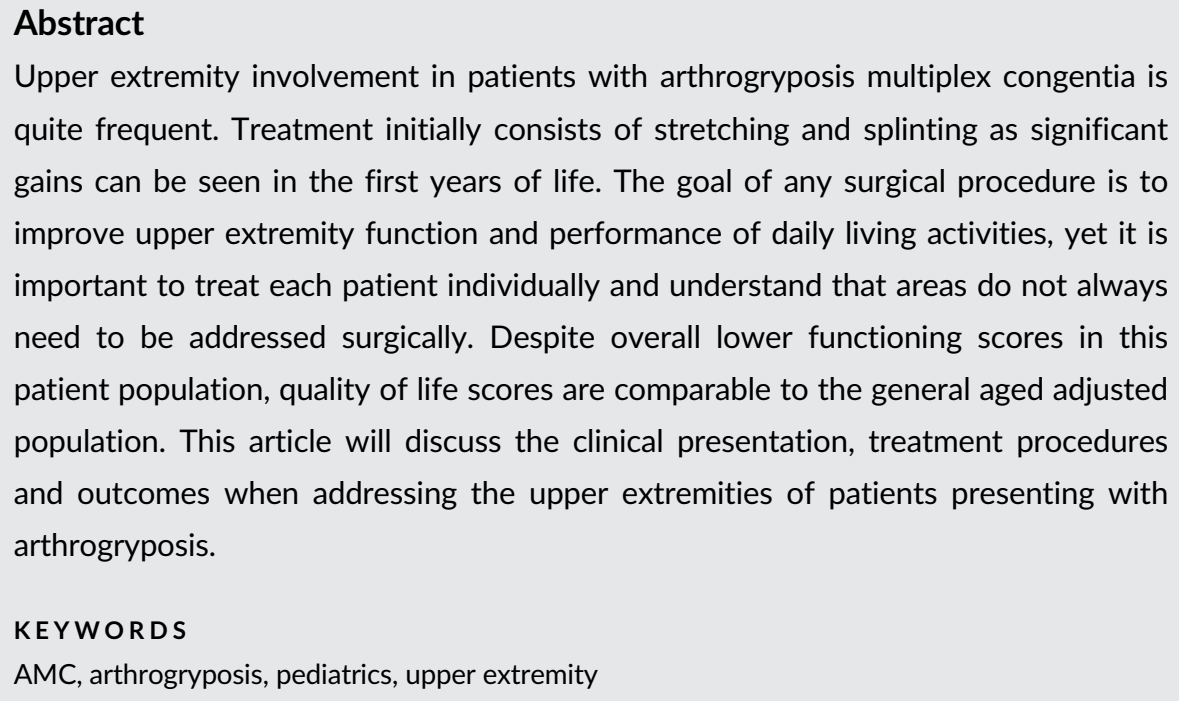

\section{1 | INTRODUCTION}

The upper extremity has frequent involvement in patients with arthrogryposis multiplex congenita (AMC), with approximately $56 \%$ of patients having upper and lower extremity involvement and 17\% having upper extremity involvement alone (Oishi et al., 2017). The "classic" position of the upper extremity at birth has been described as internal rotation of the shoulders, extension of the elbows, flexion of the wrists, thumb-in-palm deformity, and variable motion in the fingers. Despite this "classic" description wide variability is often present (Hall, 2013). It is important to note, however, that findings should be symmetric bilaterally, and any significant variation from this warrants a more proximal investigation as to potential etiology (Hall, 2014). Stretching and splinting early on is crucial, as over the first year of life there is a significant "catchup" period where large improvements may be seen (Lake \& Oishi, 2015). As a result, any consideration for surgical reconstruction should be delayed until at least 1 year of age at a minimum, and if continued improvement is still being appreciated then further delay is indicated.

Obviously, the goal of any surgical procedure is to augment upper extremity function and performance of activities of daily living (ADL's) (Abdel-Ghani, Mahmoud, Shaheen, \& Abdel-Wahed, 2017; Nouraei, Sawatzky, MacGillivray, \& Hall, 2017; Wall, Calhoun, Roberts, \& Goldfarb, 2017). Treatment must be individualized, and various approaches have been described to address the deformities. One 
important consideration for all of these patients is the significant potential anesthetic difficulties that exist (Ma \& Yu, 2017; Savenkov, Pajardi, Agranovich, Zabolskiy, \& van Bosse, 2017), and certainly surgery should only be performed in an institution that is familiar with AMC patients (Isaacson \& Drum, 2018; Savenkov et al., 2017) Although there are many surgical procedures that can be performed in these patients, it is important to only proceed if appreciable gains can be anticipated. This article will discuss both common surgical and occupational therapy treatment options for the upper extremity along with a discussion on outcome measures related to patient reported function and wellbeing. Despite each area being discussed separately, many of the procedures can be combined together, thus minimizing anesthetic exposures. It is important to note within the article is a combination of both published, evidence based practices and ideas reflecting author's clinical experiences.

\section{2 | SHOULDER}

Much research has addressed surgical treatment of the elbow, wrist, and hand, but less has focused on the shoulder internal rotation posture that is present in many of the patients. If the hands face down, either because of forearm pronation, or more commonly, shoulder internal rotation contractures, a side-to-side hand grasp is used and children either exhibit prehension with the dorsum of the wrist to the dorsum of the other wrist, or they develop crossover grasp in order to utilize palm to palm prehension. The distance they can cross over limits the size of objects they can grasp and separate their hands, which is approximately shoulder width. Also, crossing over makes transfer of objects from one place to another more difficult. Lastly, in crossover grasp the child's own arms and hands block visualization of the thumb, fingers, and palm of the hand as well as the object to be picked up and transferred.

At this time, there are only two studies that specifically address the internal rotation posturing of the shoulder (Ramirez, Richards, Kozin, \& Zlotolow, 2017; Wall et al., 2017). These two studies both advocate for humeral rotational osteotomies to address the internal rotation contracture or posture of the shoulder. Other techniques, such as release of the shoulder capsule, have been attempted but no results have been published. The concept behind humeral rotational osteotomy is to bypass the shoulder contracture and reposition the distal extremity to allow for palm to palm grasp. Because the crossover grasp pattern can result from shoulder internal rotation and/or forearm pronation contractures, it is impossible to determine the degree of shoulder or forearm rotation in an elbow that flexes less than $15^{\circ}$, therefore it is recommended to delay rotational osteotomy until after passive elbow motion has been obtained.

The surgery can be performed through a variety of approaches (medial, posterior, anterolateral, and lateral column) via a transverse osteotomy. The distal fragment is externally rotated to a degree, which will still allow for the hand to reach the mouth and midline with passive elbow flexion. The osteotomy is stabilized with a rigid plate and screw construct. The anterolateral approach is the simplest to perform and is the best choice for a more proximal diaphyseal osteotomy. While the anterolateral approach does create the most prominent scar, it does offer the simplest dissection and the easiest approach from which to determine the amount of rotation required. For children with adduction contractures at the shoulder, the anterolateral approach is also the most accessible surgically. The posterior approach is the most extensile and positioning the patient can be difficult due to shoulder contractures. However, the posterior approach allows use of previous scars from prior posterior elbow release surgery if present. We use either the posterior or the lateral column approaches when the patient requires a distal closing wedge osteotomy as well as rotational correction in order to reorient the arc of motion of the elbow in either more flexion or extension. The medial approach gives access to the mid-diaphysis and conceals the scar, but requires more dissection and technical skill, carries slightly greater risks of nerve and vessel injury, and is difficult to access in most patients. With rigid fixation, immobilization is not necessary and immediate motion is started; strengthening is begun once the osteotomy is healed.

Because of the high fall risk and disuse osteopenia commonly seen in this population, peri-implant fractures are a concern and may warrant prophylactic hardware removal in some patients. If a postosteotomy humeral fracture does occur, the shoulder can be placed into a shoulder spica cast if an adequate closed reduction can be achieved with the patient under anesthesia, or otherwise it is treated with revision of fixation. Another potential concern is recurrence of the internal rotation contracture, which is unpredictable and poorly understood. If this occurs revision osteotomy may be required. While the risk of fracture or recurrence is concerning, the potential functional gain of palm to palm prehension is arguably worth the potential complications in select patients.

\section{ELBOW}

The primary goal of elbow intervention is to allow hand to mouth reach for eating as well as preserve ability to reach the perineum for hygiene, which requires at least $90^{\circ}$ of passive flexion with minimal flexion contracture. Each child is born with different amounts of passive elbow flexion and therefore must be addressed individually. During the first year of life, stretching and splinting is an integral part of treatment that can achieve the desired $90^{\circ}$ of passive elbow flexion in some cases. In patients with minimal passive elbow flexion at initial encounter, it is imperative that proper instruction be given to parents by an experienced therapist as it can be difficult at times to know where the proper arc of motion resides, and certainly inappropriate stretching can do harm to these patients due to the abnormal anatomy and high susceptibility of joint contractures during growth (Figure 1).

At about 1 year of age, the patient should be evaluated for progress. In patients that are close to $90^{\circ}$ of passive elbow flexion, continued stretching and nighttime splinting is indicated. In the patients that have fallen significantly short of this, operative elbow release should be considered. 
FIGURE 1 Patient showing passive elbow range of motion and stretching exercises
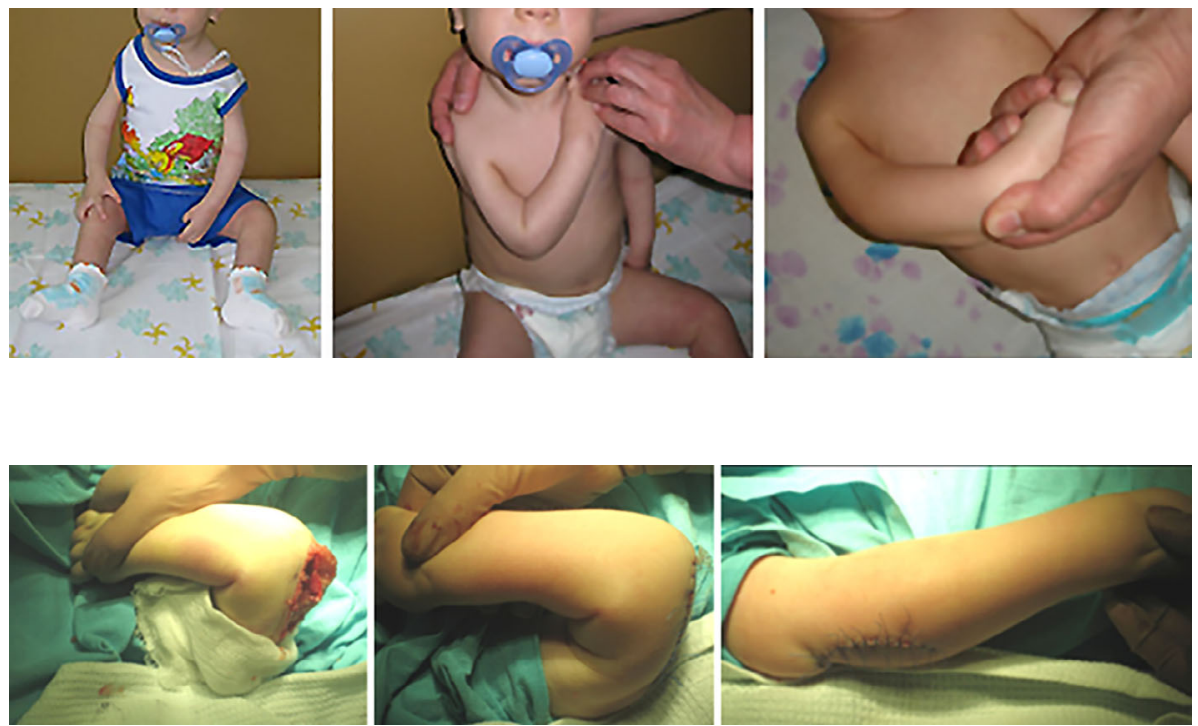

FIGURE 2 Elbow flexion

demonstrated intra and postoperatively

Elbow release is performed through a lazy $\mathrm{S}$ incision starting over the cubital tunnel and extending proximally. The ulnar nerve is carefully identified and protected, and then a W-plasty or other type of tendon lengthening procedure is fashioned over the tendinous portion of the triceps. A posterior elbow capsulotomy is then performed and the elbow is gently manipulated until the desired $90-100^{\circ}$ of passive flexion is obtained. At that point, the tendon lengthening is completed, the ulnar nerve is transposed anteriorly and the skin flaps are closed in a layered fashion (Figure 2). It is convenient to perform simultaneous wrist and thumb surgery at the same setting (if indicated), to minimize anesthetic exposure. Additionally, it is useful to perform a dressing change under anesthesia 3 weeks after the initial surgery; this concurrently allows the elbow to be manipulated under anesthesia to check for range of motion with an elbow flexion splint being fabricated. At this time, passive range of motion exercises may begin. The patient needs to be carefully watched over the next 6 months for either loss of passive elbow flexion or development of significant elbow flexion contracture. If the latter occurs, then institution of night-time elbow extension splinting is warranted.

Outcomes from this procedure have been reported in the literature with promising results. Axt et al. reported on 22 elbows in 16 children with an average follow-up of 12 years, 4 months. Postoperative passive elbow flexion averaged $88^{\circ}$ compared to $30^{\circ}$ preoperatively, with elbow flexion contracture of $49^{\circ}$ compared to $10^{\circ}$ preoperatively (Axt, Niethard, Doderlein, \& Weber, 1997). Van Heest et al. reported on three "adaptive mechanisms" that patients that obtain passive elbow flexion use to get their hands to the mouth: Table push, trunk thrust, and cervical bending. With table push, the children bend their elbows by pushing their forearms against the table at which they are seated to bring their hands to their mouths. With trunk thrust, the patients swing their arms into an elevated position by swaying their trunk and using gravity to bend their arms. With cervical bending, the children would bend their necks and use their mouths to manipulate objects or to eat food directly off the table (Van Heest, Waters, \& Simmons, 1998) (Figure 3).

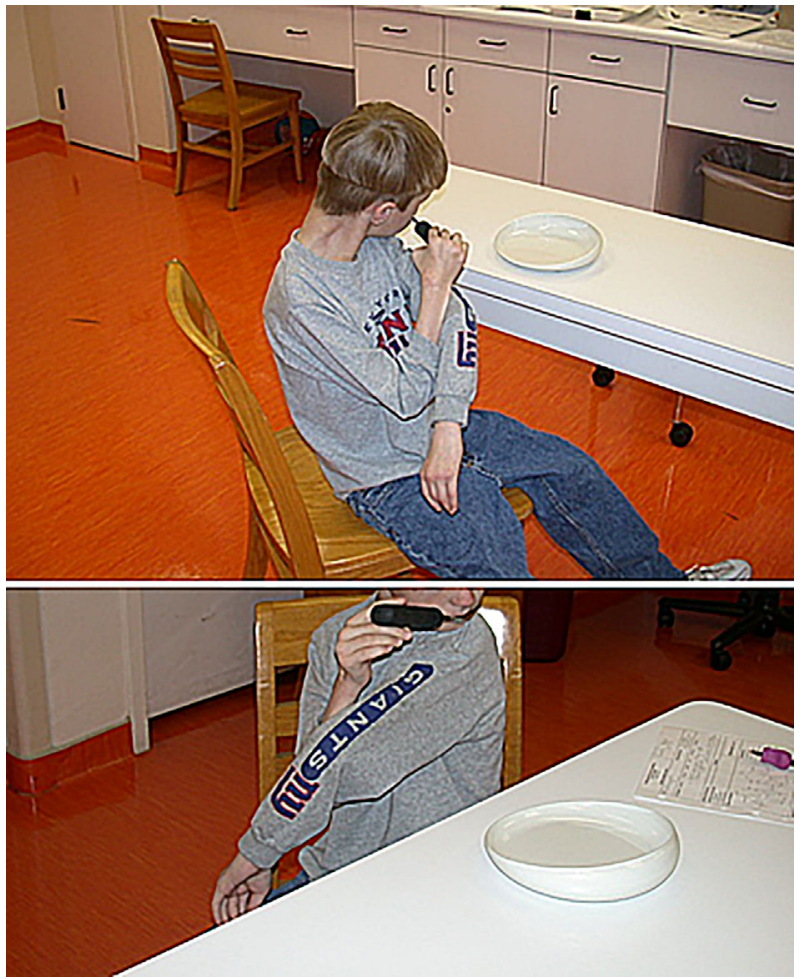

FIGURE 3 Patient who underwent an elbow release and triceps lengthening procedure demonstrating hand-to-mouth reach

Select patients can be evaluated for reconstruction of active elbow flexion. It is imperative for a comprehensive evaluation before proceeding as patients with significant elbow flexion contractures should avoid surgery as it may result in increased flexion contracture that would actually further impair overall function. In addition, the patient must be able to cooperate and participate in post-operative therapy that involves splinting and tendon transfer protocols. The ideal candidate has full passive elbow flexion and extension with some active shoulder elevation. This latter point is key, as it complements elbow flexion in getting the 


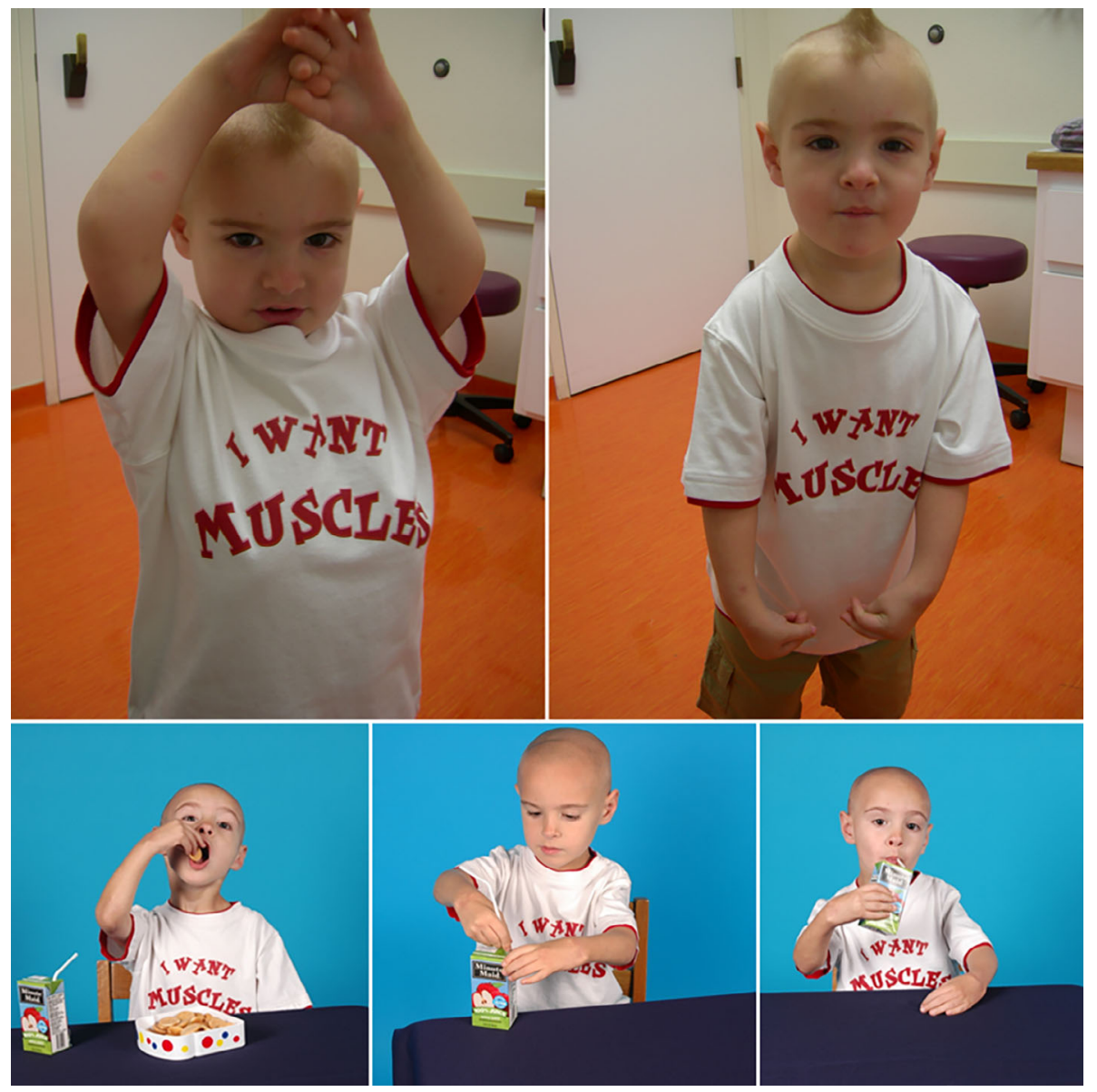

FIGURE 4 Patient who has undergone long head triceps transfer for active elbow flexion. Top are preoperative and bottom postoperative hand to the mouth. The patient must also have an expendable donor. In the back/ trunk region this can be the long-head of triceps, latissimus dorsi, or pectoralis major muscle. In the young patient, clinical examination of these muscles may be difficult and an MRI is very useful to ascertain the development and characteristics of these muscles.

The triceps muscle must have a well-developed long head and lateral/ medial heads on MRI to be considered as a donor muscle. Transfer of the entire muscle is contraindicated, as this will surely result in a severe elbow flexion contracture. The long head has an independent innervation so is well-suited for transfer as long as well-developed lateral/ medial heads are present to preserve elbow extension. The technique has been reported previously (Oishi et al., 2017) with variations on insertion of the tendon based on surgeon preference (Figure 4). The latissimus dorsi muscle is also a good alternative for tendon transfer, but careful evaluation of the MRI is imperative to assess adequate muscle development and bulk. The pectoralis major muscle can also be utilized as a tendon transfer effectively, but again MRI is imperative to assess the quality and size of the muscle. Major drawbacks of this transfer are technical difficulty related to transferring both the origin and insertion of the muscle, as well as the significant chest scarring/ breast asymmetry that results from the surgery.

After surgery, the elbow is immobilized for 3-4 weeks followed by gravity eliminated active exercises and protective splinting (Lake \& Oishi, 2015). After 8 weeks, active and passive exercises against gravity are instituted. Careful observation of these patients for development of flexion contractures is mandatory, and if this develops alternating nighttime flexion splinting with extension splinting is indicated. This is particularly true in patients that previously underwent elbow release/ triceps lengthening. It should be noted that long-term mild flexion contracture is not to be unexpected, $10-20^{\circ}$. In cases where tendon transfers are not available, free functional muscle transfer, that is, gracilis muscle can be considered. Again, an MRI is warranted to assess the presence and size of the muscle. Downsides of this reconstruction are the technical difficulties associated with microvascular surgery as well as adequacy of recipient motor nerve.

In summary, obtaining passive elbow flexion of at least $90^{\circ}$ is extremely important in most patients to facilitate hand-to-mouth activities. However, the importance of maintenance of elbow extension for perineal care and crutch use must not be ignored. If stretching and splinting is not successful during the first year of life elbow release/ triceps lengthening is a reliable option. In selected patients, muscle transfer for active elbow flexion can be useful and greatly enhance function and independence.

\section{4 | WRIST}

Wrist involvement in amyoplasia is variable in terms of the limitations in motion and function. In some children little to no intervention may 
be necessary. In those with more severely affected wrists, the common deformity is one of wrist flexion and ulnar deviation. This deformity is problematic for function in the midline when utilizing typical palm-to-palm grasp, as it places the thumb and fingers in a divergent position when attempting bimanual activities. It also poses difficulty with single-handed hand-to-mouth activities, as thumb and fingers are angled away from the face with elbow flexion. Treatment of the wrist dysfunction involves correction of the deformity while attempting to preserve as much motion as possible.

Clinical evaluation of the wrist in amyoplasia involves evaluation of the limb function as an entire unit. Shoulder, elbow, thumb, and finger function can all affect decision-making when considering interventions for the wrist. The wrist evaluation begins with measurements of active and passive wrist range of motion in both sagittal and coronal planes. In addition, the examiner should observe the child's grasp pattern and functional use with both unimanual and bimanual tasks. Radiographic evaluation of the wrist often reveals the presence of carpal coalitions between proximal and distal rows. Further evaluation with advanced imaging techniques is rarely necessary.

In infancy, the utilization of stretching and splinting can be quite successful in improving overall wrist positioning and function. Custom splints placing the wrist in progressive positions of extension and radial deviation are often utilized during nap and nighttime to assist in deformity correction. Daytime activities include passive stretching and functional use exercises. When passive stretching and splinting are unsuccessful in obtaining an acceptable wrist position, surgical intervention can be considered.

Surgery is most often reserved for those children that cannot achieve neutral wrist position in both sagittal and coronal planes through non-operative stretching and splinting protocols. The surgical procedure utilized is dependent on the degree of deformity. For children who possess active wrist extension to neutral, release of the volar fascia with lengthening or tenotomy of the wrist flexors can often allow the child to extend the wrist past neutral. This is usually coupled with transfer of the extensor carpi ulnaris (ECU) tendon to the extensor carpi radialis brevis (ECRB) dorsally to mitigate the ulnar deviation deforming force and focus the extensor power to a neutral line of pull. For patients who cannot achieve neutral wrist position by active or passive means, the use of a carpal wedge osteotomy (Ezaki \& Carter, 2004) with a volar release and ECU tendon transfer, can achieve a more functional wrist position. The carpal wedge osteotomy is performed through a transverse incision on the dorsum of the wrist at a level just distal to the radiocarpal joint. After protecting the dorsal extensor tendons to the wrist, fingers and thumb, the wrist capsule is opened transversely. A biplanar wedge osteotomy is planned with the proximal cut perpendicular to the long axis of the forearm and the distal cut perpendicular to the long axis of the metacarpals. This creates a wedge that is widest radially in the coronal plane and widest dorsally in the sagittal plane, thus correcting the flexed, ulnarly deviated position of the wrist. Care is taken to place the wedge distal to the radiocarpal joint to preserve inherent radiocarpal motion. After removal of the biplanar wedge, the osteotomy is collapsed using wrist extension and radial deviation. It is then fixed with transosseous

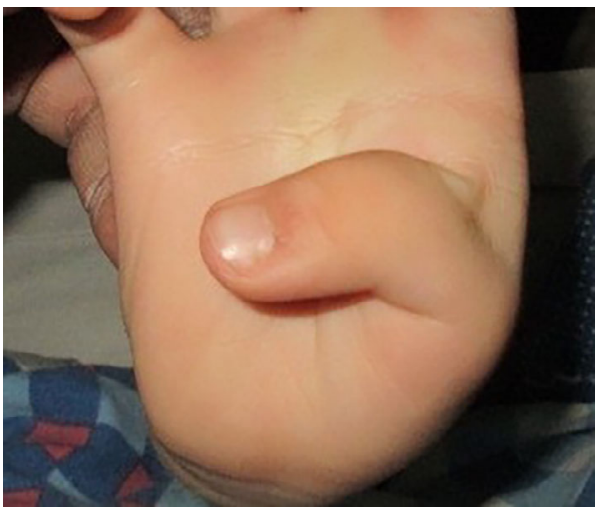

FIGURE 5 Congenital clasped thumb in patient with arthrogryposis

sutures and a K-wire for a period of 6 weeks. After k-wire removal, a removable wrist splint is used for the next 6 months.

The results following carpal wedge osteotomy have been reported by several authors in the literature. Foy et al reported on 75 wrists in 47 patients with an average of 5.7 years follow-up. Using multiple outcome measures, patients reported a mean overall satisfaction of 9.1 out of a possible 10 points (Foy, Mills, Wheeler, Ezaki, \& Oishi, 2013). They reported significant improvement $(p<.001)$ in wrist position, with no significant change in total arc of motion. In 2013, Van Heest and Rodriguez reported on 20 wrists in 12 patients. Significant increases in wrist extension (mean, $43^{\circ}$ ) were observed with no significant changes in total arc of wrist motion. In addition, the authors found significantly greater improvement in those children operated on after the age of 7 and in those who underwent concomitant ECU tendon transfer (Van Heest \& Rodriguez, 2013). Finally, De Oliveira et al found similar improvements in wrist position with little change in total arc of motion following carpal wedge osteotomy (Oliveira et al., 2018).

In patients with recurrent wrist flexion deformity following carpal wedge osteotomy, growth modulation may be an option in the skeletally immature patient.

\section{5 | CLASPED THUMB}

Clasped thumb is a characteristic deformity of the hand in patients with both AMC and distal Arthrogryposis (Figure 5). Typically, there is a lack of metacarpophalangeal joint (MPJ) extension, narrowing of the first web space with associated skin deficiency, contracture of the muscles and fascia occupying the web, and occasionally an unstable MPJ (Figure 6) and contracture of the flexor pollicis longus (FPL). In some patients, the deformity can be so severe that the thumb occupies the palm and blocks grasping. Treatment aims at restoring thumb extension and clearance of the thumb out of the palm to improve grasp. This is achieved by release of the tight, contracted structures of the web, skin augmentation of the widened web space, stabilization of the unstable MPJ by chondrodesis (if indicated), and augmentation of extensor function if appropriate. 


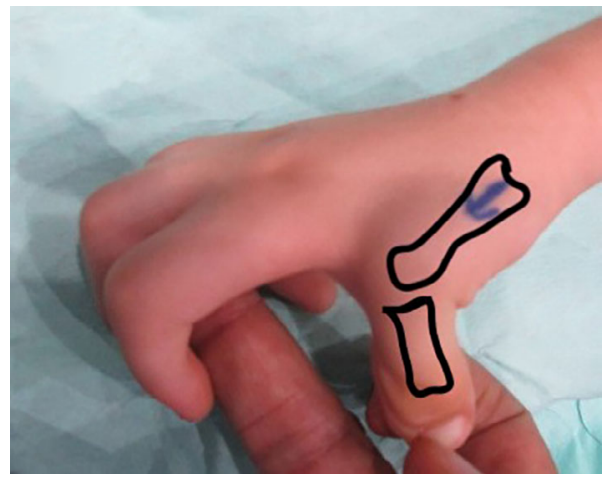

FIGURE 6 Severe adduction of the first metacarpal bone with marked narrowing of the first web space and instability of the MPJ that may give false impression of wide web. MPJ, metacarpophalangeal joint

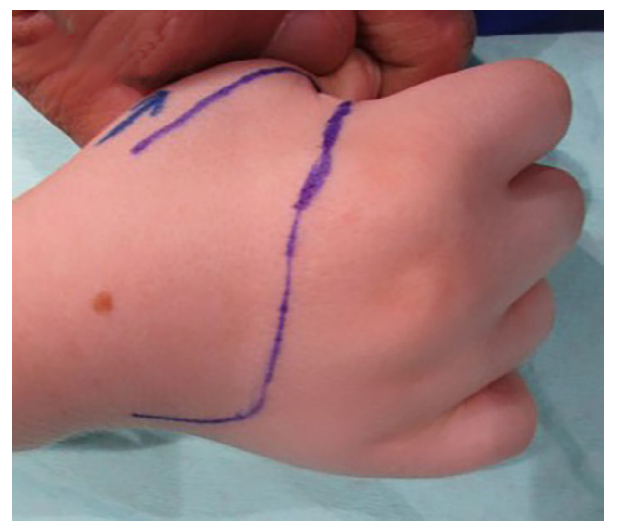

FIGURE 7 The flap is pedicled on the dorsum of the hand and extend to the edge of the web with a wide rectangular apex

One approach is to perform all the surgical steps through the skin incision of the modified dorsal rotation advancement flap (AbdelGhani Flap) (Ghani, 2006) (Figures 7 and 8) that is used to augment the skin of the web after release, although different flap designs can be utilized for skin augmentation. The tight structures of the web are released to achieve adequate palmar abduction of the thumb metacarpal. If indicated, the MPJ is released and chondrodesed in extension. In the presence of severe palmar contractures, shortening of the first metacarpal can be done at the site of chondrodesis to aid in achieving MPJ extension.

In one study, (Abdel-Ghani et al., 2017) surgery was performed on 69 complex clasped thumbs in 39 patients at a single center who were operated on at an average age of 30 months (22-36 months). At an average follow-up of 4 years (3-8 years), all parents were satisfied with the outcome with significant improvement of thumb position and function. Of note, release of the first web space involved release of the intermetacarpal fascia in all 69 thumbs, adductor pollicis in 41 thumbs, first dorsal interosseous in 35 thumbs, and CMC joint in 30 thumbs. Nonunion of the chondrodesis occurred in seven thumbs with two exhibiting fair stability and five with poor stability. The authors of the study prefer chondrodesis over soft tissue

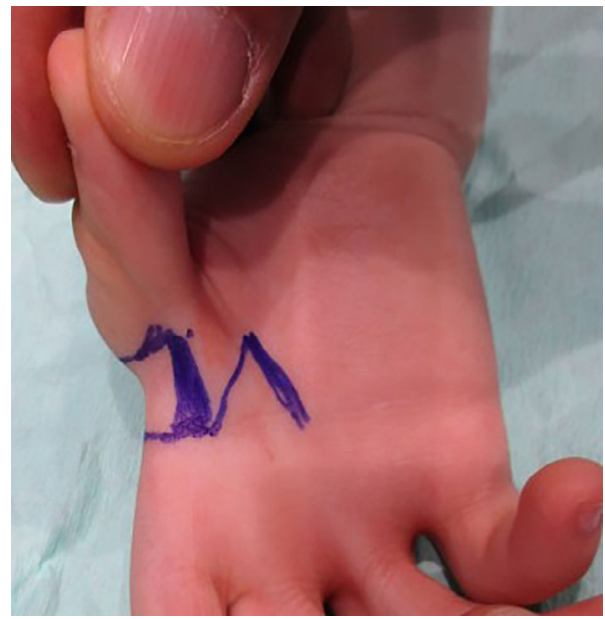

FIGURE 8 On the palmar aspect, the incision extends as Z-plasty ending at the mid palm along the axis of the middle finger

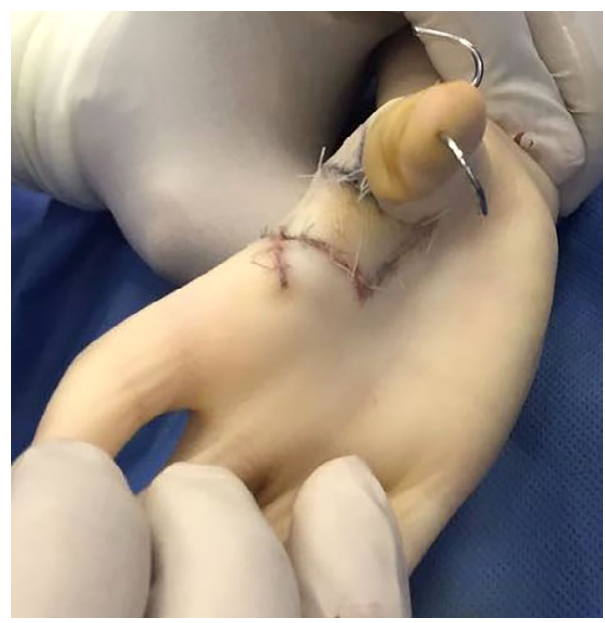

FIGURE 9 Intra operative appearance after reconstruction

reconstruction and tendon transfers because of the presence of global instability in many of the thumbs, abnormal joint structures, abnormal articular surfaces, and insufficient muscles available for transfer (Figures 9 and 10).

\section{6 | PALM AND FINGERS}

The clinical appearance of the arthrogrypotic hand can vary significantly and can include multiple camptodactylies, flexion or extension contractures of the metacarpals, windswept appearance of the fingers and palmar tightness. A thorough assessment of the deformities present is essential as to properly address the causative factors appropriately (Figures 11 and 12). As in other areas of the upper extremity, splinting and stretching exercises should be commenced shortly after birth. A close relationship of patient, parent, therapist and surgeon will ensure good compliance of a stretching and splinting regime, which may need to continue throughout the child's growing years (Babik et al., 2016; McCormick, Poling, Portillo, \& Chamberlain, 2015). 


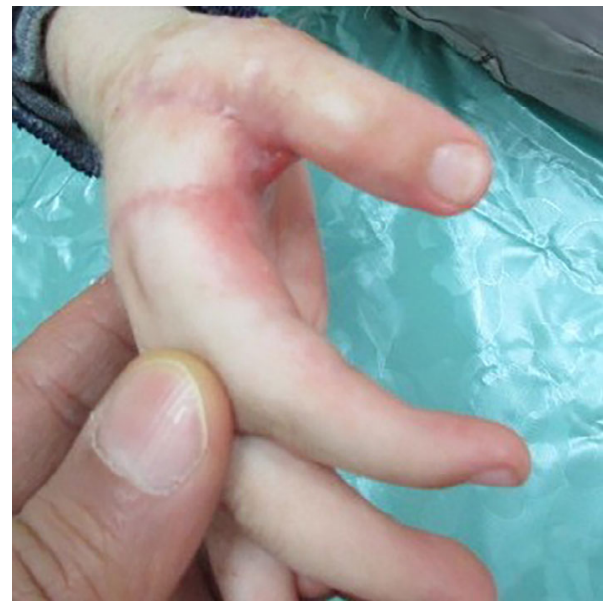

FIGURE 10 Postoperative appearance after thumb reconstruction

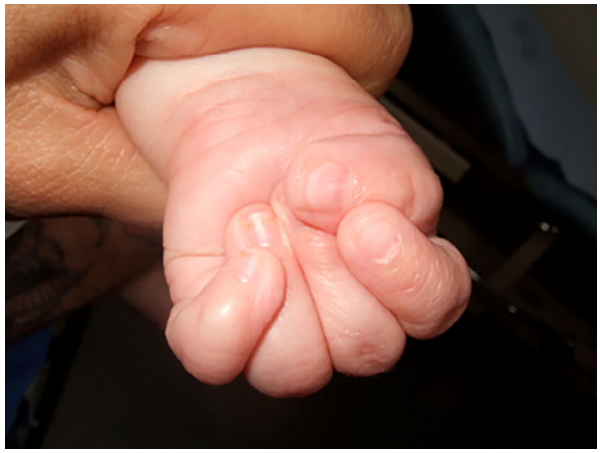

FIGURE 11 Early neonatal appearance of the hand in AMC. AMC, arthrogryposis multiplex congenita

Camptodactyly in arthrogryposis often involves a shortage of skin in both the fingers and palm. In addition, there is usually a shortening of tendons as well as bony deformities of the joints. The release of skin and soft tissue using local transposition flaps, Z-plasties (Figure 13) and full thickness grafts will address the skin deficiencies, but may not significantly address the whole contracture. In some patients, this improves cosmesis and function without attempting a full straightening of a finger. In order to fully attempt soft-tissue correction of the deformity the underlying shortening of flexor tendons as well as lack of extensor power may need to be corrected at the same time. Bony correction by osteotomy of a phalanx is rarely appropriate in the fingers. However, fusions of PIP joints, which are severely flexed and with a small range of movement, may be helpful in improving the alignment of the cascade of the hand and reduce the encumbrance of a finger. The range of movement of the arthrogrypotic finger joint can rarely be increased, but the arc of movement can be changed. It is important to note that the ability to flex a finger is more important functionally than obtaining a straightened but less mobile joint. Despite addressing all the factors listed above recurrence of the deformity to some degree is the norm, and secondary surgery may be necessary. It is important to discuss options for surgery and therapy as well as the limitations of surgery

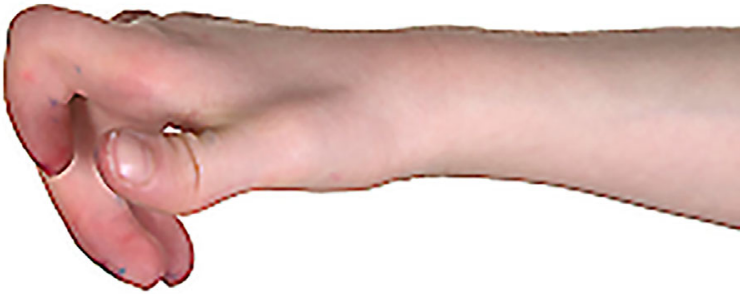

FIGURE 12 Hand in AMC with significant flexion contracture index finger extending into palm. AMC, arthrogryposis multiplex congenita

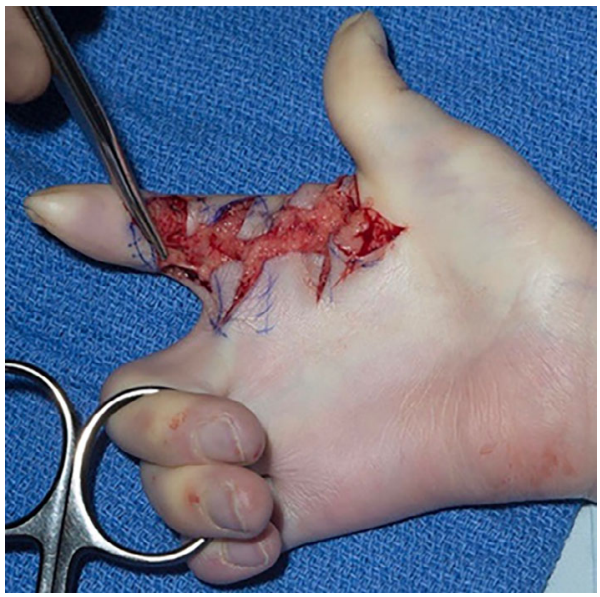

FIGURE 13 Multiple Z-plasties

with parents and/or the patients in order to deliver realistic expectations and goals.

\section{7 | OUTCOMES}

In order to assess all aspects of functioning for people with arthrogryposis, clinicians should evaluate the domains of body structure and function, activities, and participation, as described in the World Health Organization (WHO) International Classification of Function-Children and Youth (ICF-CY) (WHO, 2007). Although patients with arthrogryposis involving the upper extremity commonly have impaired motion and strength due to contractures (internal rotation at the shoulders, extension at the elbow and flexion at the wrist with variable finger flexors), they develop compensatory movements to achieve a higher level of function than may perhaps be anticipated (Dubousset \& Guillaumat, 2015). Impairment measures (such as range of motion and strength) provide information about physical function, but they do not necessarily characterize the experience of the patient regarding their ability to perform activities and participate in life roles (the activity and participation domains of the WHO ICF-CY). 
A few of the common patient reported outcome measures used to understand the changes in health for pediatric patients include the Pediatric and Adolescent Outcome Data Collection Instrument (PODCI) (Daltroy, Liang, Fossel, \& Goldberg, 1998), the Pediatric Qualify of Life Scale (PedsQL) (Varni, Seid, \& Rode, 1999), and more recently the Patient Reported Outcome Measure Information System (PROMIS) developed by the National Institute of Health (Cella et al., 2007; Riley et al., 2010). These validated measures have been utilized in the treatment of upper extremity ailments to assess the overall function, physical, mental, and social ailments of the patient (Amor, Spaeth, Chafey, \& Gogola, 2011; Bell et al., 2017; James, Abarca, \& Manske, 2018; Lerman, Sullivan, Barnes, \& Haynes, 2005; Wall et al., 2017).

Two preliminary studies show that the PROMIS Short Form tools are sensitive to evaluating upper extremity function for children with arthrogryposis. The PROMIS short form includes 5-8 questions that measure a single domain of health, for example Upper Extremity Function or Peer Relationships. The patients' responses to the questions in a single domain are converted to a $\mathrm{T}$ score for that domain, which is standardized to a population mean of 50 with a standard deviation of 10. A higher score indicates that the patient has a greater amount of the construct being tested. For domains such as Upper Extremity Function, this indicates a greater amount of a positive attribute (function); for negative attributes, such as Pain Interference, a higher score indicates greater levels of pain interference with activities.

Twenty-nine Children with arthrogryposis who are participating in the Congenital Upper Limb Differences (CoULD) registry ${ }^{1}$ had significantly lower PROMIS Upper Extremity Function scores than the general population (children with amyoplasia have an average score of 22 , almost three $S D$ s below the general population and indicating severe impairment.) PROMIS Pain Interference, Depression, Anxiety and Peer Relationships scores were within normal range for those with amyoplasia (L, CA, S, DS, \& C, 2018).

In the second study, a convenience sample of 21 children with amyoplasia who presented to a single clinic over an 8 month period in 2017 had Upper Extremity Function scores averaging 30 (moderate to severe impairment); and Pain Interference and Peer Relationships scores averaging 50 and 58, respectively (both within normal limits) (James et al., 2018).

Both studies appear to concur with clinicians' impressions that, in general, children with arthrogryposis are not severely limited by pain nor do they have substantial problems with depression, anxiety or peer relationships. These studies also support the findings of a survey of adults with amyoplasia that indicated that their quality of life is comparable to the general population (Nouraei et al., 2017). PROMIS tools are potentially useful to evaluate the impact of interventions on patient reported function for children with arthrogryposis.

\footnotetext{
${ }^{1}$ The CoULD registry is an ongoing prospective cohort study implemented in June 2014 by two U.S. tertiary-care pediatric centers and has now been expanded to include multiple centers across the United States. The purpose of the registry is to describe the epidemiology, clinical characteristics, function and health status of children with congenital upper limb differences, and to quantify changes over time with nonoperative and surgical care.
}

\section{8 | SUMMARY}

In summary, the upper extremity in patients with AMC should initially be treated with stretching and splinting as significant gains can be seen during the first year of life. At a year of age, or older if improvement has not plateaued, patients should be evaluated for possible surgery. It is important, however, to treat each patient individually, understanding that all areas do not always need to be addressed surgically. It is very common to perform elbow release, wrist, thumb, and finger surgery at the same time thus minimizing anesthetic exposures. Patients and parents must have the commitment and ability to participate in therapy or else deformities and limitations may actually worsen after surgery. It must be understood that despite overall lower function scores in these patients quality of life scores are comparable to the general population and they are generally are not limited because of pain or issues such as depression, anxiety, or peer relationships.

\section{CONFLICT OF INTEREST}

The authors declare no potential conflicts of interest with respect to the research, authorship, and/or publication of this article.

\section{AUTHOR CONTRIBUTIONS}

S.O. was involved in data collection, study design, manuscript drafting and revision. O.A., D.Z., L.W., C.S., G.P., C.N., H.A.G., A.J., C.V., M.J., M.C.M., T.B. were involved in data collection, study design and manuscript drafting.

\section{ORCID}

Scott Oishi (D) https://orcid.org/0000-0002-6581-8191

\section{REFERENCES}

Abdel-Ghani, H., Mahmoud, M., Shaheen, A., \& Abdel-Wahed, M. (2017). Treatment of congenital clasped thumb in arthrogryposis. The Journal of Hand Surgery, European Volume, 42(8), 794-798. https://doi.org/10. $1177 / 1753193417712863$

Amor, C. J., Spaeth, M. C., Chafey, D. H., \& Gogola, G. R. (2011). Use of the pediatric outcomes data collection instrument to evaluate functional outcomes in arthrogryposis. Journal of Pediatric Orthopedics, 31 (3), 293-296. https://doi.org/10.1097/BPO.0b013e31820cad93

Axt, M. W., Niethard, F. U., Doderlein, L., \& Weber, M. (1997). Principles of treatment of the upper extremity in arthrogryposis multiplex congenita type i. Journal of Pediatric Orthopaedics. Part B, 6(3), 179-185.

Babik, I., Kokkoni, E., Cunha, A. B., Galloway, J. C., Rahman, T., \& Lobo, M. A. (2016). Feasibility and effectiveness of a novel exoskeleton for an infant with arm movement impairments. Pediatric Physical Therapy, 28(3), 338-346. https://doi.org/10.1097/pep.0000000000000271

Bell, B., Butler, L., Mills, J., Stutz, C., Ezaki, M., \& Oishi, S. (2017). "On-top plasty" for radial polydactyly reconstruction. Journal of Hand Surgery, 42 (9), 753 e751-753 e756. https://doi.org/10.1016/j.jhsa.2017.05.029

Cella, D., Yount, S., Rothrock, N., Gershon, R., Cook, K., Reeve, B., ... PROMIS Cooperative Group. (2007). The patient-reported outcomes measurement information system (promis): Progress of an nih roadmap 
cooperative group during its first two years. Medical Care, 45(5 Suppl 1), S3-S11. https://doi.org/10.1097/01.mlr.0000258615.42478.55

Daltroy, L. H., Liang, M. H., Fossel, A. H., \& Goldberg, M. J. (1998). The POSNA pediatric musculoskeletal functional health questionnaire: Report on reliability, validity, and sensitivity to change. Pediatric Outcomes Instrument Development Group. Pediatric Orthopaedic Society of North America. Journal of Pediatric Orthopaedics, 18(5), 561-571.

Dubousset, J., \& Guillaumat, M. (2015). Long-term outcome for patients with arthrogryposis multiplex congenita. Journal of Children's Orthopaedics, 9(6), 449-458. https://doi.org/10.1007/s11832-015-0692-6

Ezaki, M., \& Carter, P. R. (2004). Carpal wedge osteotomy for the arthrogrypotic wrist. Techniques in Hand \& Upper Extremity Surgery, 8 (4), 224-228.

Foy, C. A., Mills, J., Wheeler, L., Ezaki, M., \& Oishi, S. N. (2013). Long-term outcome following carpal wedge osteotomy in the arthrogrypotic patient. The Journal of Bone and Joint Surgery. American Volume, 95(20), e150. https://doi.org/10.2106/jbjs.l.01122

Ghani, H. A. (2006). Modified dorsal rotation advancement flap for release of the thumb web space. Journal of Hand Therapy, 31(2), 226-229. https://doi.org/10.1016/j.jhsb.2005.10.004

Hall, J. G. (2013). Arthrogryposes (multiple congenital contractures). In D. Rimoin, R. Pyeritz, \& B. Korf (Eds.), Emery and rimoin's principles and practice of medical genetics (pp. 1-101). Oxford: Academic Press.

Hall, J. G. (2014). Arthrogryposis (multiple congenital contractures): Diagnostic approach to etiology, classification, genetics, and general principles. European Journal of Medical Genetics, 57(8), 464-472. https://doi. org/10.1016/j.ejmg.2014.03.008

Issacson, G., \& Drum, E. T. (2018). Difficult airway management in children and young adults with arthrogryposis. World Journal of Otorhinolaryngology Head and Neck Surgery, 4(2), 122-125. https://doi.org/10.1016/j. wjorl.2018.04.003

James, M., Abarca, N., \& Manske, M. (2018). Patient reported outcomes for children with arthrogyposis multiplex congenita. Paper presented at the 3rd International Symposium on Arthrogryposis, Philadelphia, PA.

Wall, L., Goldfarb, C. A., Summer, R., Bae, D. S., \& Vuillermin, C. (2018). Could Registry: Patient reported outcomes in arthrogryposis. Paper presented at the 3rd International Symposium on Arthrogryposis, Philadelphia, PA.

Lake, A. L., \& Oishi, S. N. (2015). Hand therapy following elbow release for passive elbow flexion and long head of the triceps transfer for active elbow flexion in children with amyoplasia. Journal of Hand Therapy, 28(2), 222-226; quiz 227. https://doi.org/10.1016/j.jht. 2014.10.007

Lerman, J. A., Sullivan, E., Barnes, D. A., \& Haynes, R. J. (2005). The pediatric outcomes data collection instrument (podci) and functional assessment of patients with unilateral upper extremity deficiencies. Journal of Pediatric Orthopedics, 25(3), 405-407.

Ma, L., \& Yu, X. (2017). Arthrogryposis multiplex congenita: Classification, diagnosis, perioperative care, and anesthesia. Frontiers in Medicine, 11 (1), 48-52. https://doi.org/10.1007/s11684-017-0500-4

McCormick, R. J., Poling, M. I., Portillo, A. L., \& Chamberlain, R. L. (2015). Preliminary experience with delayed non-operative therapy of multiple hand and wrist contractures in a woman with freeman-Sheldon syndrome, at ages 24 and 28 years. BML Case Reports, 2015, 1-5. https:// doi.org/10.1136/bcr-2015-210935

Nouraei, H., Sawatzky, B., MacGillivray, M., \& Hall, J. (2017). Long-term functional and mobility outcomes for individuals with arthrogryposis multiplex congenita. American Journal of Medical Genetics. Part A, 173 (5), 1270-1278. https://doi.org/10.1002/ajmg.a.38169

Oishi, S. N., Agranovich, O., Pajardi, G. E., Novelli, C., Baindurashvili, A. G., Trofimova, S. I., ... Butler, L. (2017). Treatment of the upper extremity contracture/deformities. Journal of Pediatric Orthopedics, 37(Suppl 1), S9-S15. https://doi.org/10.1097/bpo.0000000000001002

de Oliveira, R. K., da Silva Marques, F., Praetzel, R. P., Bayer, L. R., Delgado, P. J., \& Ribak, S. (2018). Biplanar carpal wedge osteotomy in the treatment of the arthrogrypotic patients. Revista Brasileira de Ortopedia, 53(6), 687-695. https://doi.org/10.1016/j.rboe.2017.08.026

Ramirez, R. N., Richards, C. J., Kozin, S. H., \& Zlotolow, D. A. (2017). Combined elbow release and humeral rotational osteotomy in arthrogryposis. The Journal of Hand Surgery, 42(11), 926 e921-926 e929. https://doi.org/ 10.1016/j.jhsa.2017.06.005

Riley, W. T., Rothrock, N., Bruce, B., Christodolou, C., Cook, K., Hahn, E. A., \& Cella, D. (2010). Patient-reported outcomes measurement information system (promis) domain names and definitions revisions: Further evaluation of content validity in irt-derived item banks. Quality of Life Research, 19(9), 1311-1321. https://doi.org/10.1007/s11136-010-9694-5

Savenkov, A. N., Pajardi, G. E., Agranovich, O. E., Zabolskiy, D., \& van Bosse, H. J. P. (2017). Anaesthesiology for children with arthrogryposis. Journal of Pediatric Orthopedics, 37(Suppl 1), S27-S28. https://doi.org/10. 1097/bpo.0000000000000998

Van Heest, A., \& Rodriguez, R. (2013). Dorsal carpal wedge osteotomy in the arthrogrypotic wrist. The Journal of Hand Surgery, 38(2), 265-270. https://doi.org/10.1016/j.jhsa.2012.10.034

Van Heest, A., Waters, P. M., \& Simmons, B. P. (1998). Surgical treatment of arthrogryposis of the elbow. The Journal of Hand Surgery, 23(6), 1063-1070. https://doi.org/10.1016/s0363-5023(98)80017-8

Varni, J. W., Seid, M., \& Rode, C. A. (1999). The PedsQL: Measurement model for the pediatric quality of life inventory. Medical Care, 37(2), 126-139.

Wall, L. B., Calhoun, V., Roberts, S., \& Goldfarb, C. A. (2017). Distal humerus external rotation osteotomy for hand position in arthrogryposis. The Journal of Hand Surgery, 42(6), 473 e471-473 e477. https://doi.org/10. 1016/j.jhsa.2017.03.002

WHO. (2007). World health organization: International classification of functioning, disability and health: Children and youth (pp. 1-351), Geneva: World Health Organization.

How to cite this article: Oishi S, Agranovich O, Zlotolow D, et al. Treatment and outcomes of arthrogryposis in the upper extremity. Am J Med Genet Part C. 2019;181C:363-371.

https://doi.org/10.1002/ajmg.c.31722 